\title{
¿Puede el neuromarketing ayudar a mejorar el diseño de la web de compra? Un estudio neurocientífico sobre entornos de compra hedónicos y utilitarios
}

\author{
Luis-Alberto Casado-Aranda \\ Profesor ayudante doctor. Universidad de Granada (España) \\ Icasado@go.ugr.es | https://orcid.org/0000-0002-2725-492X \\ Juan Sánchez-Fernández \\ Catedrático de universidad. Universidad de Granada (España) \\ sanchezf@ugr.es | https://orcid.org/0000-0001-6725-920X
}

Este trabajo ha obtenido el 1.er Premio Estudios Financieros 2021 en la modalidad de Marketing y Publicidad.

El jurado ha estado compuesto por: don Ubaldo Cuesta Cambra, doña Alicia Izquierdo Yusta, doña Esmeralda López Alonso, doña Sonia San Martín Gutiérrez y don Francisco Javier Sesé Olivan.

Los trabajos se presentan con seudónimo y la selección se efectúa garantizando el anonimato de los autores.

\section{Extracto}

\begin{abstract}
Investigaciones previas en el campo del e-commerce han definido los dos entornos web más ampliamente utilizados, a saber, el hedónico (entorno visualmente atractivo, centrado en el disfrute e interactivo) y el utilitario (informativo y funcional). No obstante, no existen conclusiones unánimes sobre la influencia de dichas tipologías en el recuerdo por parte del consumidor. Además, ningún estudio ha evaluado los mecanismos psicológicos que subyacen al procesamiento de los sitios web hedónicos y utilitarios, que podrían ser cruciales, dado el origen inconsciente e introspectivo de las decisiones de compra online. Esta investigación utiliza por primera vez la neuroimagen (resonancia magnética funcional, fMRI) para identificar diferencias neuronales entre el recuerdo de sitios web hedónicos y utilitarios. Los datos neuronales esclarecen el origen del mayor recuerdo hacia los entornos hedónicos y confirman que, mientras que los diseños hedónicos activan áreas cerebrales relacionadas con la emoción, codificación de la memoria y la memoria de trabajo, los sitios web utilitarios solo activan redes cerebrales relacionadas con la memoria de trabajo y función deliberativa. En su conjunto, estos resultados aportan información crucial sobre la conexión de los datos neuropsicológicos con el comportamiento del consumidor online, y pueden ayudar al diseño de entornos web eficientes.
\end{abstract}

Palabras clave: neurociencia del consumidor; entorno web; hedónico; utilitario; comportamiento del consumidor.

Cómo citar: Casado-Aranda, L.-A. y Sánchez-Femández, J. (2021). ¿Puede el neuromarketing ayudar a mejorar el diseño de laweb de compra? Un estudio neurocientífico sobre entornos de comprahedónicos yutilitarios. Revista de Marketing y Publicidad. CEF, 4, 7-30. https://doi.org/10.51302/marketing.2021.800 


\title{
Can neuromarketing help improve website layout? A neuroscientific study on hedonic and utilitarian shopping environments
}

\author{
Luis-Alberto Casado-Aranda \\ Juan Sánchez-Fernández
}

\section{Abstract}

Previous research in the e-commerce field has defined the two most widely used web environments, namely the hedonic (visually appealing environment, focused on the hedonic (focused and interactive) and utilitarian (informative and functional). Yet, there are no unanimous conclusions on the influence of these typologies on consumer recall. Moreover, no study has evaluated the psychological mechanisms underlying the processing of hedonic and utilitarian websites, which could be crucial, given the unconscious and introspective origin of online purchase decisions. The current reasearch uses neuroimaging (functional magnetic resonance imaging, fMRI) for the first time to identify neural differences between hedonic and utilitarian website recall. The neural data shed light on the origin of enhanced recall for hedonic environments and confirm that, while hedonic designs activate brain areas related to emotion, memory encoding and working memory, utilitarian websites only elicit brain networks related to working memory and memory function. Taken together, these results provide crucial information about the connection of neuropsychological data to online consumer behavior, and may help in the design of efficient web environments for potential consumers.

Keywords: consumer neuroscience; web environment; hedonic; utilitarian; consumer behavior.

Citation: Casado-Aranda, L.-A. y Sánchez-Fernández, J. (2021). ¿Puede el neuromarketing ayudar a mejorar el diseño de la web de compra? Un estudio neurocientífico sobre entomos de compra hedónicos y utilitarios. Revista de Marketing y Publicidad. CEF, 4, 7-30. https://doi.org/10.51302/marketing.2021.800 


\section{Sumario}

1. Introducción

2. Antecedentes teóricos y justificación del estudio

2.1. ¡Las características del sitio web importan! Sitios web hedónicos y utilitarios

2.2. Procesamiento de sitios web hedónicos y utilitarios

2.3. Neurociencia del consumidor y e-commerce: Respuestas neuronales a los sitios web hedónicos y utilitarios

2.3.1. El valor de la neuroimagen en los entornos de e-commerce

2.3.2. La presente investigación: Mecanismos neuronales del recuerdo de páginas web hedónicas y utilitarias

3. Metodología

3.1. Participantes

3.2. Diseños de páginas web

3.3. Procedimiento

3.4. Análisis de datos de neuroimagen

4. Resultados

4.1. Resultados comportamentales

4.2. Respuestas neuronales a los sitios web hedónicos y utilitarios

5. Discusión

6. Limitaciones, futuras investigaciones

7. Conclusiones

Referencias bibliográficas 


\section{Introducción}

En los últimos años, el sector del comercio electrónico (e-commerce, en adelante) ha crecido exponencialmente. En 2020, las ventas internacionales en el canal online crecieron un 27,6 respecto a 2019, ascendiendo a 4,280 billones de dólares (eMarketer, 2021). Las ventas en e-commerce, de hecho, representaron el 16,1\% del total de las ventas minoristas mundiales en 2020 y se espera que alcancen el $22 \%$ en 2023 . Mientras que en 2016 el 58,3\% de los usuarios internacionales de internet había comprado productos online, en 2020 esta cifra creció hasta el 64,6 \% (Statista, 2021). Con este crecimiento exponencial, la distribución online de tecnología, entretenimiento/cultura y alimentación ha ido en aumento, consolidándose como las principales categorías de productos comprados en 2020 en España, escenario del presente estudio (eMarketer, 2021).

En consecuencia, el canal de e-commerce es cada vez más competitivo, y los minoristas electrónicos están invirtiendo grandes cantidades de recursos en la selección del entorno web más eficaz que mejore las experiencias de los consumidores y promueva la compra de sus productos. La literatura emergente sobre marketing directo e interactivo corrobora que los entornos web (como la interfaz de usuario, el entretenimiento, los colores y la estructura del sitio) constituyen los elementos más críticos a la hora de influir en los estados emocionales del consumidor durante la compra online, y estos, a su vez, afectan a las actitudes de los compradores, a la eficacia percibida de la web, a los niveles de satisfacción y al comportamiento del consumidor. De hecho, en España, el $74 \%$ de los compradores busca información y formaliza una compra basándose únicamente en las características y datos incluidos en el canal online (eMarketer, 2021).

Dada la importancia de las características del sitio web en el proceso de evaluación y decisión de compra de productos online, investigaciones anteriores han examinado cómo las diversas tipologías de características del sitio web afectan a las actitudes, emociones y comportamientos de los consumidores (Moon et al., 2017). Estos estudios corroboran que dicha influencia no es necesariamente consciente, sino que se produce a nivel interno, implícito e introspectivo (Mazaheri et al., 2012). De hecho, las investigaciones han demostrado que los procesos psicológicos, afectivos y cognitivos internos derivados de la percepción de los entornos online determinan la fidelidad a la compra en la web, la confianza en el producto de un e-retailer (Casado-Aranda et al., 2018) e incluso la tasa de clics en el sitio web (Couwenberg et al., 2017).

Los estudios sobre las respuestas internas a las características del sitio web se han implementado en gran medida utilizando técnicas de autoinforme (es decir, cuestionarios sobre 
las actitudes de los consumidores, las intenciones o la eficacia percibida del sitio web), que, sin embargo, han demostrado ser insuficientes para capturar las emociones más internas que ocurren durante la toma de decisiones de los consumidores. Por ejemplo, las metodologías de autoinforme por sí solas no son capaces de captar las reacciones del consumidor en cada momento, de identificar el origen de estados psicológicos como la confianza, el riesgo o la recompensa hacia las características del sitio web y están sujetas a sesgos como la deseabilidad social (Motoki et al., 2020). Dado el papel crucial de los procesos internos en la determinación de la evaluación del producto en los diseños de compra online, se necesitan herramientas complementarias que registren una medición más precisa, objetiva e instantánea de tales mecanismos.

En este sentido, las técnicas de neurociencia del consumidor se están consolidando en el ámbito del e-commerce como herramientas de enorme valor para proporcionar una comprensión más rica, imparcial e interna del origen de la toma de decisiones del consumidor (Casado-Aranda et al., 2019). En el presente estudio, utilizamos, por primera vez, la neuroimagen, concretamente la resonancia magnética funcional (fMRI), con el objetivo de (1) explorar las diferencias neuronales que ocurren durante el recuerdo de dos tipologías de sitios webs: hedónicos y utilitarios.

\section{Antecedentes teóricos y justificación del estudio}

\section{1. iLas características del sitio web importan! Sitios web hedónicos y utilitarios}

La literatura sobre el comportamiento del consumidor ha evaluado ampliamente qué factores fomentan la compra de productos en las tiendas físicas y ha esclarecido que el merchandising visual, la comodidad de la ubicación, la actitud y la experiencia del personal de ventas y el precio (Guerrero Medina et al., 2020) constituyen algunos de los elementos más importantes en la formación de las actitudes del consumidor y las intenciones de compra en el canal físico. En el mercado online, la ausencia de estas interacciones interpersonales físicas ha sido sustituida por la interacción electrónica y tecnológica, y las actitudes y los comportamientos son impulsados por las características del diseño del sitio web. En las dos últimas décadas, la investigación sobre marketing interactivo ha demostrado que las decisiones de compra online se basan en gran medida en el diseño y la apariencia del sitio web, incluidas las fotos, las características de facilidad de uso, la calidad de la información o los enlaces a las redes sociales. Una revisión bibliográfica (Rose et al., 2011) concluyó que las pistas y características del entorno web constituyen insumos que afectan directamente a las decisiones de compra. De hecho, se ha demostrado que las evaluaciones positivas de un sitio web conducen a una actitud más positiva, a la intención e incluso a la compra de bienes específicos. 
Dada la importancia de las características del sitio web en la configuración de la intención y el comportamiento de compra, la literatura sobre e-commerce ha intentado clasificar los elementos que los minoristas electrónicos incluyen en sus sitios web para identificar el efecto de cada categoría en la toma de decisiones del consumidor. Por ejemplo, Mazaheri et al. (2012) distinguieron entre elementos de alta y baja relevancia para la compra. El primer grupo incluye descriptores que facilitan directamente la consecución de la compra, como la calidad y cantidad de información, la funcionalidad, la accesibilidad, la rapidez y la estructura del sitio web. Por su parte, el segundo incorpora elementos que no son cruciales para completar la compra online, como el color del texto, el tamaño de los gráficos y la música. Autores como Liang y Lai (2002) identificaron tres categorías: factores motivacionales, como la inclusión de un motor de búsqueda eficiente y el apoyo directo al consumidor durante el proceso de compra; factores higiénicos, como proporcionar una buena seguridad y proteger a los consumidores de los riesgos; y medios de comunicación, como proporcionar salas de chat y añadir más canales de información al proceso. Posteriormente, Hausman y Siekpe (2009) consideraron que el canal online engloba algunos elementos de naturaleza funcional/informática y otros de naturaleza humana. Los elementos informáticos son aquellos cuya presencia proporciona funcionalidad, como los aspectos técnicos, la imparcialidad y el contenido de la información. Los factores humanos añaden valor a la web al contribuir a la satisfacción del usuario, como el disfrute, la capacitación del usuario, el aspecto visual y la credibilidad. Más recientemente, Bilgihan y Bujisic (2015) distinguieron entre factores utilitarios y hedónicos. El primer grupo se centra en el diseño y el aumento del valor y la funcionalidad del diseño web mediante la accesibilidad, la capacidad de búsqueda de información y la provisión de atributos claros del producto. La segunda clasificación incluye la diversión, la fantasía y el aumento de la emoción del comprador y está orientada a las actividades de ocio. Los elementos hedónicos se refieren a imágenes animadas, colores, sonidos, enlaces a redes sociales, avatares y entornos web atractivos.

En conjunto, los elementos estudiados anteriormente dan lugar a dos tipos de sitios web de compras: (1) los sitios web utilitarios, que incluyen predominantemente características que requieren evaluaciones racionales de los consumidores, la identificación y el reconocimiento del producto y proporcionan un apoyo directo, funcional e informativo al proceso transaccional y a las características y beneficios del producto; y (2) los sitios web hedónicos, que incorporan mayoritariamente factores poco relevantes para la compra y promueven el disfrute del consumidor, la autorrelevancia, las experiencias visuales y la interactividad asociada al producto. Mientras que un sitio web utilitario incluiría información detallada, precisa y sin color sobre el producto vendido, un sitio web hedónico apenas incorpora información sobre el producto, pero añade color, avatares o imágenes usando el producto.

La literatura sobre e-commerce ha evaluado recientemente, sin consenso, cuál de estos dos tipos de sitios web es más eficaz para promover las compras online. El estudio realizado por Rosen y Purinton (2004) demostraron que los estímulos sensoriales, como los 
elementos hedónicos, desempeñan un papel crucial a la hora de facilitar la usabilidad y promover la repetición de las compras. Hausman y Siekpe (2009) descubrieron que ofrecer medios más ricos en un contexto más real (es decir, sitios web hedónicos) «tiene una influencia más positiva en la implicación del usuario con la compra que los factores utilitarios». Por su parte, Bilgihan y Bujisic (2015) concluyeron que tanto los factores hedónicos como los utilitarios son indispensables para generar confianza en un sistema online, así como para la fidelización. Más concretamente, los autores revelan que los elementos hedónicos construyen un compromiso afectivo, es decir, un apego emocional que permite a los consumidores desarrollar una relación positiva con el sitio web. Los factores utilitarios, por su parte, son cruciales para desarrollar un compromiso calculador, es decir, la intención de continuar la relación con la empresa, teniendo en cuenta los costes y la falta de alternativas.

Otros estudiosos han afirmado, sin embargo, que la eficacia de las características del sitio web depende principalmente del propio producto. En particular, indican que las características deben coincidir con el tipo de producto; es decir, un sitio web utilitario es más apropiado para bienes utilitarios (por ejemplo, microondas), mientras que un sitio web hedónico es más eficaz para bienes hedónicos (por ejemplo, chocolate) (Motoki et al., 2020). En otras palabras, «los consumidores que compran productos utilitarios tienden a preferir canales de información que permitan realizar búsquedas y comparaciones cómodas y eficientes de los atributos de los productos", mientras que la naturaleza experiencial de las compras hedónicas se ajusta más a los canales hedónicos, caracterizados por un menor número de páginas de productos e información visual (Li et al., 2020). Otros estudios indican incluso que «en una categoría de productos que no es claramente hedónica ni utilitaria, no hay diferencias significativas en la motivación de búsqueda de variedad cuando la variación proviene de atributos sensoriales o funcionales» (Baltas et al., 2017).

\subsection{Procesamiento de sitios web hedónicos y utilitarios}

Dado que las investigaciones sobre la eficacia de las características hedónicas o utilitarias de los sitios web han generado resultados ambiguos, es imprescindible aclarar cómo procesan los consumidores los sitios web hedónicos y utilitario, y si el recuerdo generado por uno u otro sitio web es similar. La literatura sobre marketing interactivo sugiere que los dos tipos de diseños web utilizan dos vías de procesamiento diferentes. Por un lado, la naturaleza visual y basada en la experiencia, la sorpresa, la aventura, la diversión y la interactividad que ofrecen los sitios web hedónicos fomentan el procesamiento emocional del sitio web. En particular, cuando se exponen a entornos hedónicos online, los consumidores se ven impulsados a confiar en pistas simples y heurísticas en lugar de un procesamiento de información más profundo y detallado para llegar a sus decisiones de compra. En consecuencia, en lugar de tratar de encontrar las alternativas y los bienes más beneficiosos, «los consumidores pueden desarrollar un apego afectivo a las marcas y los productos 
ofrecidos (Li et al., 2020) y pueden procesar la información de forma más pausada, emocional y holística». La posibilidad de incluir logotipos de redes sociales, colores y avatares de relevancia personal en los sitios web hedónicos puede desencadenar una señalización positiva de la información y una alta relevancia para el individuo y, por lo tanto, conducir a la implicación de sentimientos emocionales, gratificantes y autorreferenciales entre los consumidores online (Casado-Aranda et al., 2019; Couwenberg et al., 2017). Algunas investigaciones han confirmado, de hecho, que la apelación a lo emocional y afectivo (en relación con la información específica y datos) puede generar mejores tasas de recuerdo (Panda et al., 2013).

Por otro lado, los sitios web utilitarios facilitan que los consumidores realicen una evaluación racional y orientada a objetivos, con el objetivo de tomar la decisión de compra más óptima a partir de la información proporcionada en el sitio web. En este caso, los clientes evalúan el resultado de la compra comparando los beneficios percibidos al adquirir el producto online con los costes. Por lo tanto, los consumidores pueden desarrollar un procesamiento de la información más profundo, deliberado y planificado a través de los atributos concretos especificados en el sitio web. En esta línea, los consumidores pueden experimentar mecanismos de identificación y reconocimiento del objeto al ver el producto y leer la información relacionada. Además, los consumidores pueden experimentar el control cognitivo, es decir, un conjunto de procesos que organizan, planifican y programan las decisiones mentales de comprar, o no, un producto online.

\subsection{Neurociencia del consumidor y e-commerce: Respuestas neuronales a los sitios web hedónicos y utilitarios}

\subsubsection{El valor de la neuroimagen en los entornos de e-commerce}

Las herramientas tradicionales que se han utilizado para identificar los procesos internos de los consumidores en relación con las características de los sitios web se basan en opiniones declarativas y autoinformadas de los consumidores, principalmente encuestas, grupos de discusión y entrevistas. A pesar de que estas herramientas son relativamente baratas y fáciles de usar, se basan en el hecho de que los consumidores pueden expresar con éxito sus procesos cognitivos y emocionales internos y posteriormente utilizarlos en sus elecciones de compra online (Casado-Aranda et al., 2019). Esto es solo parcialmente cierto, ya que estas herramientas no son capaces de capturar las emociones instantáneas y de bajo orden que tradicionalmente tienen lugar durante compras online, son susceptibles a la deseabilidad social y a la subjetividad y no ofrecen fácilmente una recopilación en tiempo real de las reacciones del consumidor al ejecutar una tarea (Dimoka, 2010). Una consecuencia directa de las limitaciones de los autoinformes es la falta de posibilidad de registrar gran parte del proceso de compra online; en consecuencia, los datos de estas técnicas corren el riesgo de no explicar del todo el comportamiento del consumidor online. 
La neurociencia del consumidor o neuromarketing constituye una de las ramas del marketing con mayor potencial en la actualidad para complementar las herramientas tradicionales e identificar los orígenes implícitos de la toma de decisiones del consumidor. Mediante técnicas como la resonancia magnética funcional (fMRI), es posible acceder a los procesos cognitivos y afectivos que tienen lugar justo cuando los consumidores evalúan o toman decisiones online. En concreto, la fMRI es la técnica con mayor resolución espacial, utilidad y capacidad para evaluar en profundidad las reacciones de la estructura cerebral durante la exposición a los estímulos. Esta herramienta detecta los cambios en el nivel de oxigenación de la sangre del cerebro (es decir, la señal BOLD), que luego se utiliza como indicador de la activación neuronal. Dado que diferentes regiones de interés (ROI por sus iniciales en inglés, regions of interest) están asociadas a funciones mentales concretas (por ejemplo, recompensa, valor o riesgo), es posible, por tanto, localizar y explorar los mecanismos neuronales desencadenados por diversos diseños de sitios web y representarlos visualmente mediante imágenes cerebrales de fMRI. Por tanto, podría permitirnos identificar el origen neural implícito de la evaluación y recuerdo de los sitios web hedónicos y utilitarios (véase figura 1).

Figura 1. Valor añadido de la neurociencia del consumidor al campo del e-commerce

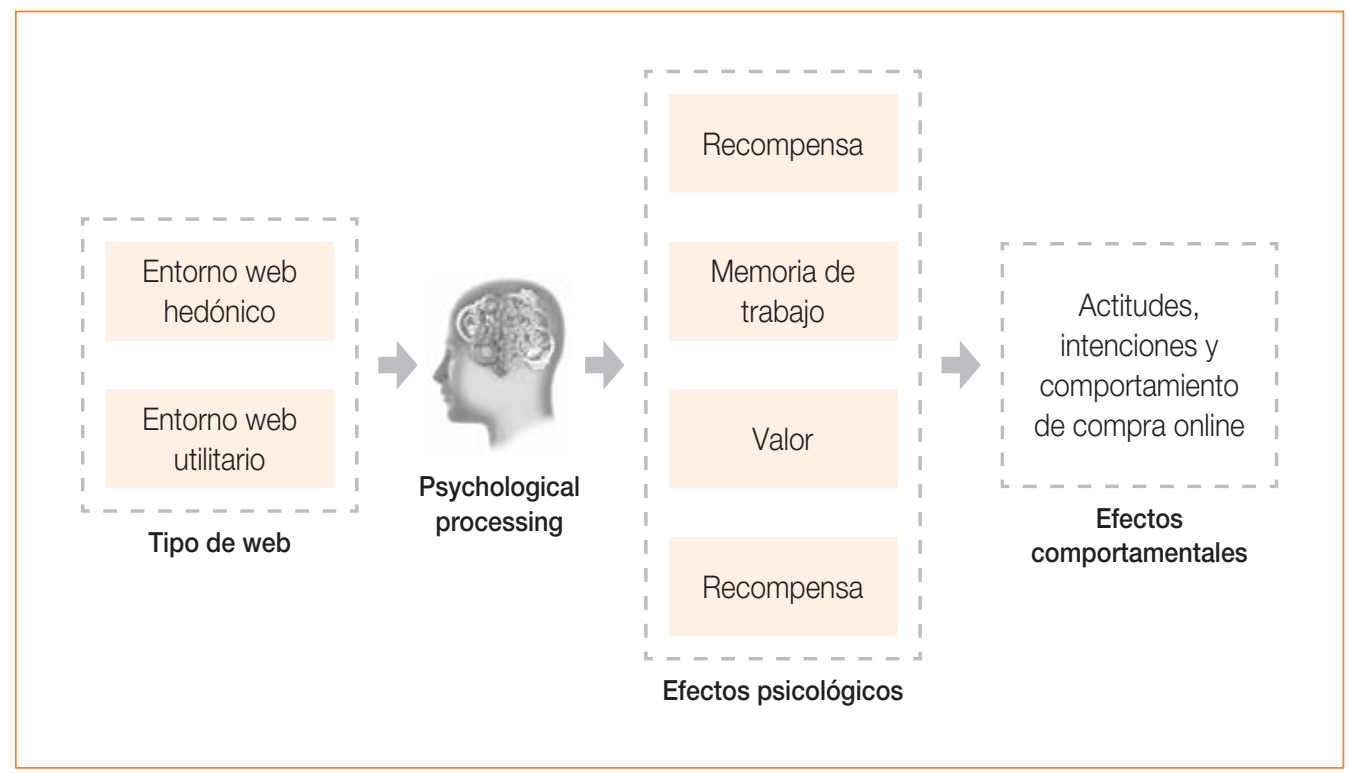

Aunque la neuroimagen tiene desventajas como la dificultad del análisis de los datos, el entorno artificial y el alto coste, los estudios recientes de fMRI han arrojado luz sobre el origen de las decisiones de los consumidores en el entorno online y proporcionan conocimientos fructíferos y complementarios a los datos de autoinforme. Por ejemplo, 
Casado-Aranda et al. (2018) analizaron el procesamiento neural de los pagos electrónicos arriesgados y seguros y concluyeron que PayPal es un medio de pago más confiable debido a las áreas relacionadas con la recompensa y la confianza que involucra en comparación con una tarjeta de débito. Curiosamente, Motoki et al. (2020) utilizaron una combinación de medidas neuronales autoinformadas y relacionadas con la sociedad para predecir el éxito del marketing viral en las redes sociales. En una línea similar, Hubert et al. (2018) hicieron uso de la fMRI para identificar cómo la impulsividad del consumidor modula el procesamiento neuronal de diversos diseños online que varían en niveles de confianza. Sin embargo, es sorprendente observar que ninguna investigación, hasta la fecha, ha analizado el origen neural de la evaluación de diferentes tipos de características de los sitios web, un hecho que arrojaría luz sobre la escasa unanimidad acerca de la eficacia de los entornos de compra online hedónicos y utilitarios.

\subsubsection{La presente investigación: Mecanismos neuronales del recuerdo de páginas web hedónicas y utilitarias}

La presente investigación contribuye a la amplia bibliografía sobre los efectos de las características de los sitios web en el comportamiento de compra en línea mediante el uso de innovadoras técnicas de neuroimagen con el objetivo de (1) comprender mejor el origen neural interno del recuerdo de sitios web hedónicos y utilitarios, y (2) evaluar cómo estas respuestas neurales se alinean con el recuerdo reportado por los consumidores sobre dichas webs.

En concreto, la literatura del comportamiento de compra online ha revelado que aquellos entornos que provocan un procesamiento emocional, de mayor valor y relevancia para el consumidor generan mejores actitudes hacia la marca y mayor recuerdo del entorno o anuncio (Falk et al., 2010; Panda et al., 2013). De hecho, el estudio de Couwenberg et al. (2017) demostró que los entornos experienciales (tales como los hedónicos) provocan una mayor codificación de la memoria, memoria de trabajo, reconocimiento y recuperación de la memoria de los elementos de comunicación expuestos, dada la evaluación más creativa y emocional que los consumidores desarrollan ante dichos entornos web. La memoria de trabajo se refiere a la parte de la memoria a corto plazo que se ocupa del procesamiento perceptivo y lingüístico consciente inmediato. Por su parte, la codificación es el acto de introducir la información en nuestro sistema de memoria a través de un procesamiento automático o con esfuerzo. Por su parte, la recuperación es el acto de sacar la información del almacén y llevarla a la conciencia mediante el recuerdo, el reconocimiento y el reaprendizaje.

En particular, la extensa literatura de neuroimagen ha concluido que la memoria de trabajo está implicada con la activación de los lóbulos temporales y frontales medios. La codificación de la memoria, en cambio, implica la activación de áreas cerebrales como el hipocampo y el lóbulo superior frontal medio (Tsukiura y Cabeza, 2011). La recuperación de la memoria ha sido ampliamente relacionada con la activación del precúneo y el giro 
parahipocampo. Por último, el reconocimiento de estímulos previamente visualizados se ha asociado con la activación del giro fusiforme y el lóbulo temporal inferior y medio. Por lo tanto, hipotetizamos que:

Áreas cerebrales implicadas con la memoria de trabajo (lóbulo temporal y frontal medio), la codificación de la memoria (hipocampo y lóbulo frontal superior), recuperación de la memoria (precúneo y giro parahipocampo) y reconocimiento (giro fusiforme y lóbulo temporal inferior y medio) se activan más fuertemente durante el recuerdo de sitios web hedónicos frente a los utilitarios.

\section{Metodología}

\subsection{Participantes}

Se reclutaron 30 participantes para el experimento de $\mathrm{fMRI}$, aunque finalmente solo se conservaron los datos de 27 participantes sanos porque dos hombres y una mujer se sintieron incómodos durante la tarea experimental. En concreto, se seleccionaron 15 mujeres diestras y 12 hombres, con una edad media de 24,30 (SD = 5.40) años. Todos los participantes dieron su consentimiento informado antes de las sesiones de fMRI, siguiendo el Comité de Ética de la Universidad de X y la Declaración de Helsinki. Debido a que el nivel de experiencia de los participantes y la frecuencia de las compras online pueden afectar a su procesamiento de los sitios web (Rose et al., 2011), solo se seleccionaron participantes con niveles similares de estas variables. En concreto, todos los participantes declararon un nivel medio de conocimientos informáticos y de la web de 5,52 (DT: 1.12) en una escala Likert de 7 puntos (anclada en 1 = pocos conocimientos y $7=$ muchos conocimientos). Además, el $100 \%$ de los participantes declaró haber comprado al menos una vez al año un producto en internet, y el $88 \%$ compró online al menos una vez al semestre y el $8 \%$ una vez a la semana.

\subsection{Diseños de páginas web}

El propósito de la tarea de fMRI era simular un contexto de compra online y recuerdo de productos tecnológicos en dos tipos diferentes de sitios web: hedónico y utilitario. En concreto, se utilizó un diseño intra-sujeto en el que cada participante debía evaluar la compra de 60 auriculares diferentes, 30 en un entorno web hedónico y 30 en uno utilitario. La elección se restringió a los auriculares porque las investigaciones de neuroimagen más recientes han utilizado esta categoría de producto (Casado-Aranda et al., 2019) y porque la tecnología, junto al entretenimiento, la cultura y la alimentación, es uno de los sectores con mayor número de ventas online en España (eMarketer, 2021). Como procedimiento de ma- 
nipulación, queríamos asegurarnos de que todos los participantes habían comprado recientemente productos tecnológicos en internet y que, además, tenían un interés e intención común de comprar auriculares. En concreto, el 73,1\% de la muestra declaró haber comprado productos tecnológicos en el último año, frente al $65,2 \%$ de la moda, el $56 \%$ de la educación y el $48 \%$ de la música. En una escala Likert de 7 puntos (donde 1 = nivel más bajo y 7 = nivel más alto), los participantes declararon un interés por comprar auriculares de una media de $5,74(S D=1,29)$ y una intención de compra de $4,70(S D=1,73)$.

Como se ha explicado anteriormente, la literatura sobre marketing interactivo confirma que el tipo de producto (es decir, preeminentemente hedónico o utilitario) puede afectar al tratamiento del producto y al diseño del sitio web en el que se presenta (Li et al., 2020). Para corroborar si los auriculares se encasillan claramente como un producto percibido como hedónico o utilitario, utilizamos las escalas hedónicas y utilitarias de las actitudes de los consumidores, tal y como informan Motoki et al. (2020) y Couwenberg et al. (2017). En concreto, se pidió a los participantes que expresaran sus opiniones en una escala Likert de 7 puntos, desde 1 (nada) hasta 7 (mucho), sobre los siguientes ítems hedónicos y utilitarios: «hedónico: divertido / no divertido, emocionante / aburrido, encantador / no encantador, emocionante / no emocionante, agradable/no agradable; utilitario: eficaz / no eficaz, útil / no útil, funcional / no funcional, necesario / no necesario, práctico / no práctico» (Motoki et al., 2020). Tras promediar las puntuaciones de cada tipología de producto, los resultados mostraron que los auriculares no pueden clasificarse como productos preeminentemente hedónicos o utilitarios, ya que no existen diferencias significativas entre las escalas hedónica (media hedónica $=5,45$; $S D=0,93$ ) y utilitaria (media utilitaria 5,79; $S D=0,53)(p=0,123$ ). En consecuencia, y siguiendo las conclusiones de Baltas et al. (2017), el tipo de producto aquí no tendrá un efecto indeseable sobre la motivación o la evaluación del diseño de la página web.

Una vez que confirmamos que los auriculares eran productos adecuados para el diseño experimental, desarrollamos 30 webs hedónicas y 30 utilitarias siguiendo las directrices de la literatura anterior sobre los efectos del diseño web en el comportamiento de compra online (Bilgihan y Bujisic 2015; Mazaheri et al., 2012). En concreto, las webs hedónicas presentaban colores, imágenes de productos, avatares y enlaces a redes sociales. En cambio, los sitios web utilitarios no incluían colores, pero presentaban información detallada sobre las ventajas y los atributos de los auriculares (véase figura 2). Una muestra independiente $(n=60)$ en una prueba preliminar corroboró los controles de manipulación de los sitios web experimentales; en una escala Likert de 7 puntos, desde 1 (sitio web informativo, conveniente y utilitario) y 7 (sitio web visualmente atractivo, centrado en la alegría, interactivo y hedónico), una prueba t de muestras emparejadas reveló diferencias significativas entre los 30 sitios web hedónicos (media hedónica $=5,23$; $S D=0,25$ ) y los 30 utilitarios (media utilitarian $=2,08 ; S D=0,36)(p<0,001)$. También se incluyó un sitio de marca ficticio (Tecnobuy) con el objetivo de evitar cualquier familiaridad o preferencia previa hacia la marca y, en consecuencia, evitar cualquier efecto de confusión en la evaluación de los sitios web. 
Figura 2. Representación de las dos tipologías de web que se mostraron a los participantes: (A) hedónica y $(B)$ utilitaria

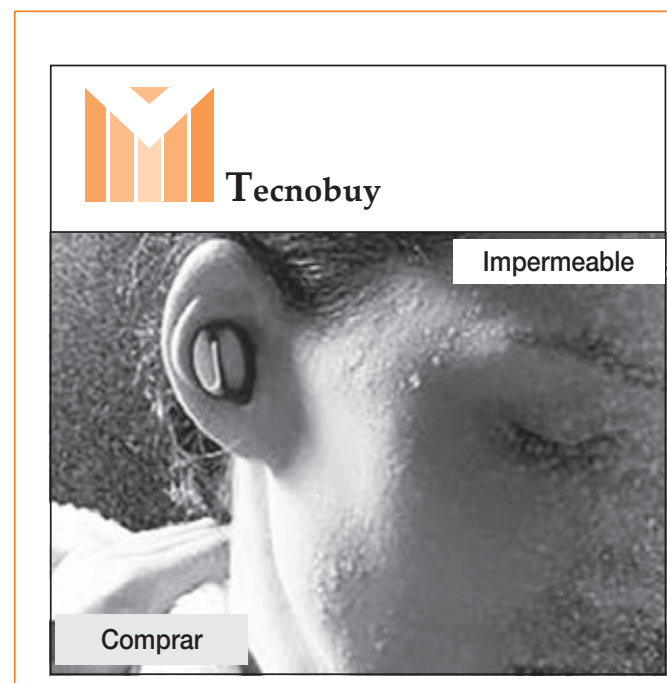

A. Web de compra hedónica

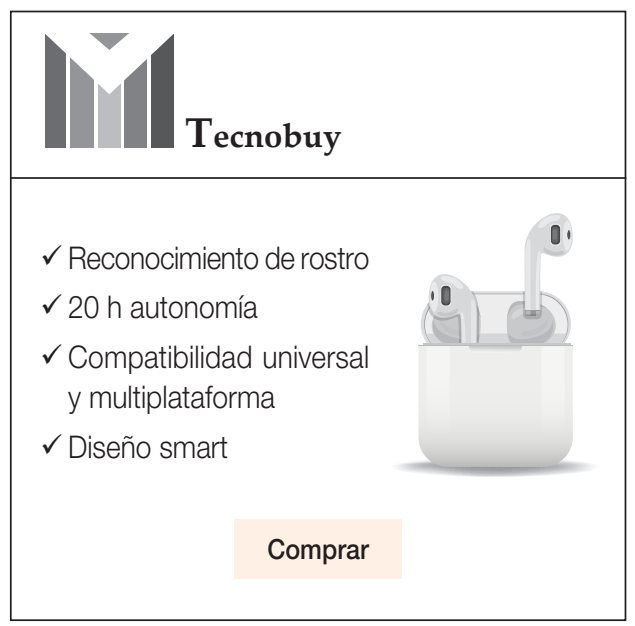

B. Web de compra utilitaria

\subsection{Procedimiento}

Los sujetos llegaron al laboratorio de fMRI una hora antes de la tarea para corroborar los datos del consentimiento informado y comprobar, de nuevo, los criterios comunes de exclusión de fMRI (por ejemplo, embarazo, implantes metálicos o claustrofobia). A continuación, se expuso a los participantes específicamente a diversos auriculares en 30 sitios web hedónicos diferentes y 30 utilitarios distintos. Cada serie de diseños de sitios web comenzaba con una visualización de un breve periodo de fijación (4 s), seguido de una visualización de $8 \mathrm{~s}$ de un sitio web hedónico o utilitario seleccionado al azar. Por último, los participantes tenían que cerrar los ojos para volver a imaginar el sitio web que habían visto anteriormente durante $6 \mathrm{~s}$. La duración total de la exploración fue de unos 24 minutos, incluido el tiempo de obtención de imágenes anatómicas. Utilizamos el software E-Prime Professional 2.0 para presentar los estímulos de fMRI (véase en la estructura de la tarea en figura 3).

Tras la tarea de fMRI, los participantes respondieron a cuestionarios informatizados autoadministrados. Concretamente, se los solicitó a los participantes que realizaran una tarea de memoria por sorpresa con el objetivo de evaluar su nivel de recuerdo de los entornos web durante la exploración. La prueba consistía en ver un total de 40 entornos web divididos en 20 hedónicos y 20 utilitarios. Cada una de los 20 entornos se dividió en 10 vistos durante el escaneado de fMRI y 10 imágenes nuevas. 
Figura 3. Estructura de la tarea de neuroimagen experimental

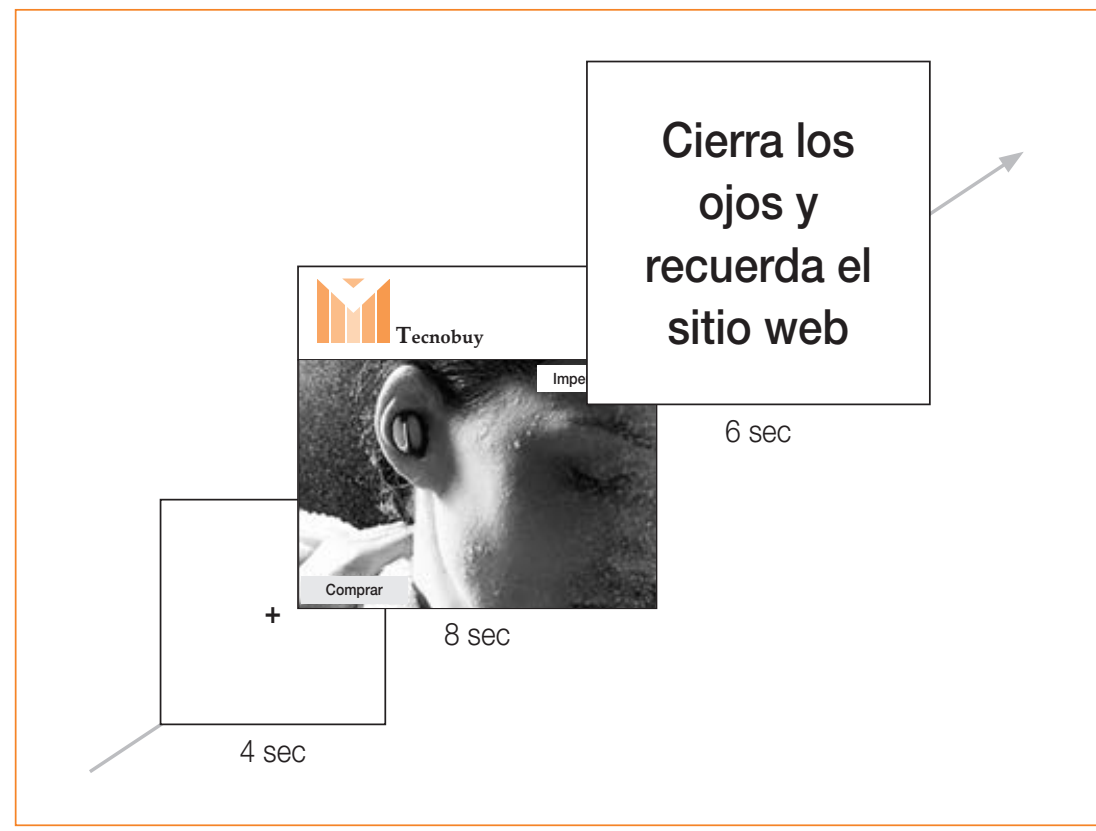

\subsection{Análisis de datos de neuroimagen}

Se utilizó un escáner Trio Siemens de 3T con una bobina de cabeza de 32 canales para obtener las imágenes de fMRI. Las imágenes anatómicas se adquirieron utilizando una orientación sagital con un tamaño de voxel de $1 \mathrm{~mm} \times 1 \mathrm{~mm} \times 1 \mathrm{~mm}$. Las exploraciones funcionales se adquirieron mediante una secuencia de imagen ecoplanar (EPI) ponderada en $\mathrm{T}^{*}$ ( $\mathrm{TR}=2000$ $\mathrm{ms}, \mathrm{TE}=25 \mathrm{~ms}, \mathrm{FA}=90^{\circ}$, grosor $=3,5 \mathrm{~mm}$; cortes $=35$, orden de los cortes $=$ descendente). $\mathrm{El}$ factor de distancia del $20 \%$ dio como resultado un total de 790 cortes con un FoV de $238 \mathrm{~mm}$.

Se preprocesaron y analizaron los datos de neuroimagen utilizando un software estándar (SPM12, Wellcome Department of Cognitive Neurology, Londres, Reino Unido, https:// www.fl.ion.ucl.ac.uk/spm/software/spm12/) ejecutado en MATLAB R2012a. Se utilizó la configuración por defecto que se aplicó en SPM. Las imágenes funcionales medias se comprobaron visualmente para detectar artefactos. A continuación, las imágenes funcionales se realinearon para corregir el movimiento, se corregistraron, se segmentaron, se normalizaron en el espacio estereotáctico estándar y se suavizaron (kernel gaussiano de $7 \times 7 \times 7 \mathrm{~mm}$ FWHM). Posteriormente, generamos mapas estadísticos para cada participante ajustando una función boxcar a las series temporales convolucionadas con una función de respuesta hemodinámica canónica. El resultado consistió en una estimación de un modelo lineal general (GLM) para cada participante que incluía los siguientes regresores de interés: 
(1) periodo de reimaginación de webs hedónicas y (2) periodo de reimaginación de webs utilitarias. Además, se utilizaron seis covariables asociadas al ruido relacionado con el movimiento, un término de sesión constante y cruces de fijación como regresores sin interés.

En el primer nivel de análisis, para identificar qué regiones cerebrales muestran diferentes activaciones en los sitios web hedónicos y utilitarios, se calcularon dos contrastes: reimaginar sitios web hedónicos vs. utilitarios (1 vs. 2) y viceversa, aplicando un contraste $T$ a los primeros y segundos regresores del modelo, respectivamente. En el segundo nivel, los contrastes de la comparación hedónico vs. utilitario (y viceversa) se introdujeron en un análisis de pruebas t de una muestra para identificar las redes de activación cerebral comunes a todos los participantes.

El presente estudio aplicó la metodología de regiones de interés (ROI) para analizar las imágenes funcionales. Se trata de un enfoque basado en la teoría que recurre a coordenadas específicas relacionadas en metaanálisis anteriores con procesos cognitivos como la recuperación o codificación de la memoria. Este análisis de ROI recurrió específicamente a la base de datos de acceso abierto Neurosynth (Yarkoni et al., 2011), que resumió metanalíticamente una gran cantidad de investigaciones de mapeo cerebral e identificó sustratos neuronales asociados a procesos cognitivos de interés para el presente proyecto: codificación de la memoria, reconocimiento, memoria de trabajo y recuperación de la memoria. Específicamente, se extrajeron cuatro máscaras de ROI específicas: 1) una máscara de procesamiento de la codificación de la memoria basada en 146 estudios que aplicaban el término codificación de la memoria para la búsqueda, 2) una máscara de procesamiento del reconocimiento basada en 1.101 estudios que aplicaban el término reconocimiento, 3) una tercera máscara basada en 1.091 estudios relacionados con la memoria de trabajo, 4) y una cuarta máscara basada en 166 estudios de autorreferencia, y 5) una máscara basada en la recuperación de la memoria basada en 228 estudios.

\section{Resultados}

\subsection{Resultados comportamentales}

Se utilizó el programa informático IBM Statistical Package of Social Science (IBM SPSS, versión 20) para realizar los análisis estadísticos. Concretamente, se diseñó una prueba de Wilkoxon para identificar las divergencias de las valoraciones de los niveles de recuerdo hacia los dos tipos de webs de compra online. Los niveles de rendimiento de la prueba de memoria sorpresa demuestran en general niveles más altos de recuerdo de las webs de compra hedónicas. Específicamente, los participantes revelaron significativamente $(p<0.001)$ una mayor precisión a la hora de identificar adecuadamente entornos webs hedónicos (proporción media correcta $=0.94 ; \mathrm{SD}=0.07$ ) en relación a los entornos utilitarios (proporción media correcta $=0.84 ; \mathrm{SD}=0.1$. 


\subsection{Respuestas neuronales a los sitios web hedónicos y uti- litarios}

A continuación, se evaluó si existen diferencias neuronales al recordar las páginas web hedónicas y utilitarias expuestas durante el experimento de fMRI. Los resultados indicaron que los sitios web hedónicos provocaron mayores activaciones cerebrales en las áreas de interés implicadas con la codificación de la memoria, reconocimiento, memoria de trabajo y recuperación de la memoria. En concreto, los diseños hedónicos provocaron la activación del hipocampo, giro fusiforme y giro superior frontal, todas ellas áreas cerebrales incluidas en la codificación de la memoria (Lin et al., 2017). El giro cingular y el lóbulo temporal inferior y medio, áreas cerebrales relacionadas con el reconocimiento (Borghesani et al., 2019), también se activaron fuertemente durante la evaluación de los sitios web hedónicos. Además, los lóbulos temporales y frontales medios, áreas implicadas con la memoria de trabajo (Johnson et al., 2018), se activaron más fuertemente ante entornos hedónicos que utilitarios. Por último, los diseños hedónicos también activaron áreas cerebrales relacionadas con la recuperación de la memoria, como el precúneo y el parahipocampo (Hebscher et al., 2019) (véanse la tabla 1 y la figura 4).

Tabla 1. Valores de las regiones cerebrales resultantes del análisis de las ROI extraídas de la base de datos www.neurosynth.org que sirven para contrastar el procesamiento de webs hedónicas vs. utilitarias

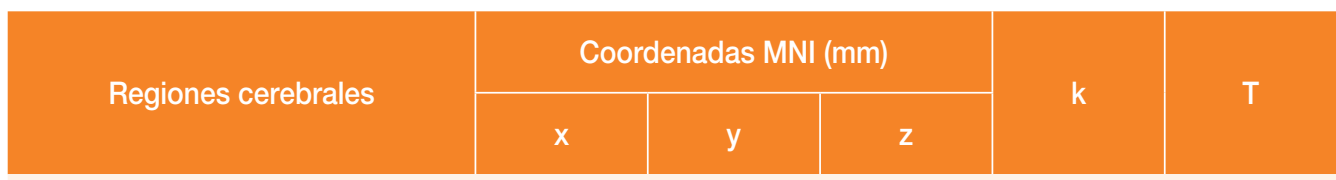

Reimaginar web hedónica vs. reimaginar web utilitaria

Codificación de la memoria

$\begin{array}{lrrrcc}\text { Hipocampo } & -20 & -32 & -4 & 20 & 7,70 \\ \text { Giro fusiforme } & 26 & -32 & -21 & & \\ \text { Hipocampo } & 29 & -14 & -18 & 19 & 5,52 \\ \text { Hipocampo } & -17 & -7 & -18 & & \\ \text { Giro superior frontal medial } & -6 & -49 & 39 & 4 & 4,26 \\ \text { Reconocimiento } & & & & & \\ \text { Giro fusiforme } & 36 & -53 & -21 & 204 & 12 \\ \text { Giro fusiforme } & -34 & -42 & -14 & 70 & 7,33 \\ \text { Giro cingular } & -10 & -39 & 32 & 7 & 4,94\end{array}$




\begin{tabular}{|c|c|c|c|c|c|}
\hline \multirow{2}{*}{ Regiones cerebrales } & \multicolumn{3}{|c|}{ Coordenadas MNI (mm) } & \multirow{2}{*}{ k } & \multirow{2}{*}{$\mathrm{T}$} \\
\hline & $x$ & $y$ & z & & \\
\hline \multicolumn{6}{|l|}{ 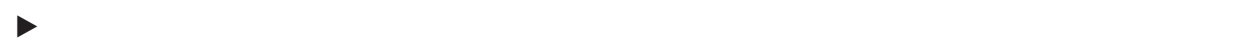 } \\
\hline Lóbulo temporal inferior & 43 & -11 & -28 & 10 & 4,79 \\
\hline Lóbulo temporal medio & -55 & -67 & 21 & 5 & 4,54 \\
\hline
\end{tabular}

Memoria de trabajo

$\begin{array}{lrrrrr}\text { Giro temporal medio } & 50 & -67 & 4 & 2 & 7,38 \\ \text { Hipocampo } & -17 & -7 & -18 & 18 & 5,42 \\ \text { Lóbulo frontal medio } & -41 & 18 & 39 & 4 & 4,87 \\ \text { Recuperación de la memoria } & 1 & -63 & 39 & 5 & 4,25 \\ \text { Precúneo } & 5 & -49 & 14 & 15 & 6,17 \\ \text { Precúneo } & 29 & -39 & -7 & 5 & 5,83 \\ \text { Parahipocampo } & & & & \end{array}$

Por el contrario, la reimaginación de los sitios web utilitarios solo provocó activaciones implicadas con la memoria de trabajo, tales como el lóbulo inferior frontal, parietal superior o giro precentral (Johnson et al., 2018) (véanse la tabla 2 y la figura 4).

Tabla 2. Valores de las regiones cerebrales resultantes del análisis de las ROI extraídas de la base de datos www.neurosynth.org que sirven para contrastar el procesamiento de webs utilitarias vs. hedónicas

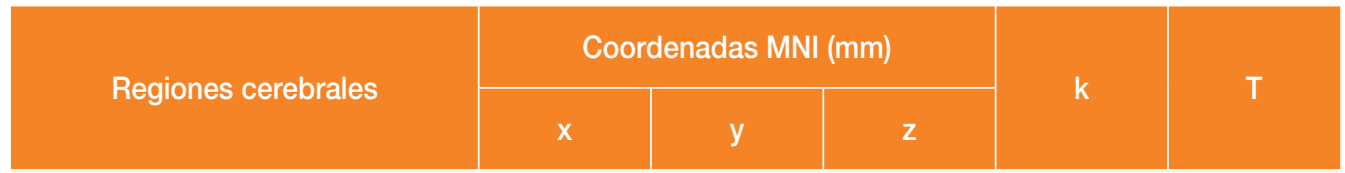

Reimaginar web utilitaria vs. reimaginar web hedónica

Codificación de la memoria 


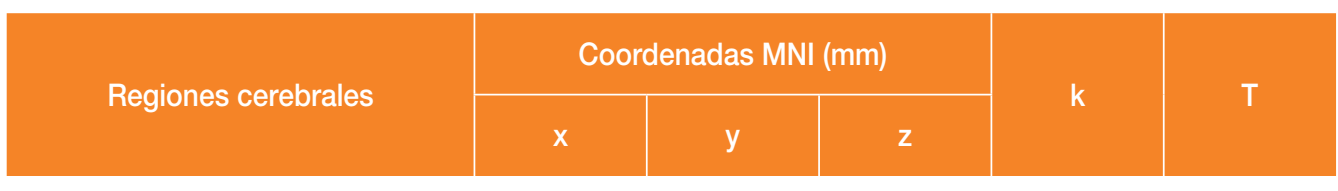

Reconocimiento

Memoria de trabajo

$\begin{array}{lrrrrr}\text { Giro precentral } & -45 & -4 & 42 & 34 & 8,14 \\ \text { Área superior motor } & -6 & 4 & 67 & 44 & 7,63 \\ \text { Lóbulo parietal superior } & -25 & -60 & 53 & 23 & 6,55 \\ \text { Lóbulo inferior frontal } & -48 & 11 & 21 & 8 & 3,83\end{array}$

Memoria de trabajo

Recuperación de la memoria

Figura 4. Regiones de interés (ROI) del cerebro derivadas de los mapas de inferencia inversa extraídos de www.neurosynth.org vinculados a la memoria de webs hedónicas y utilitarias

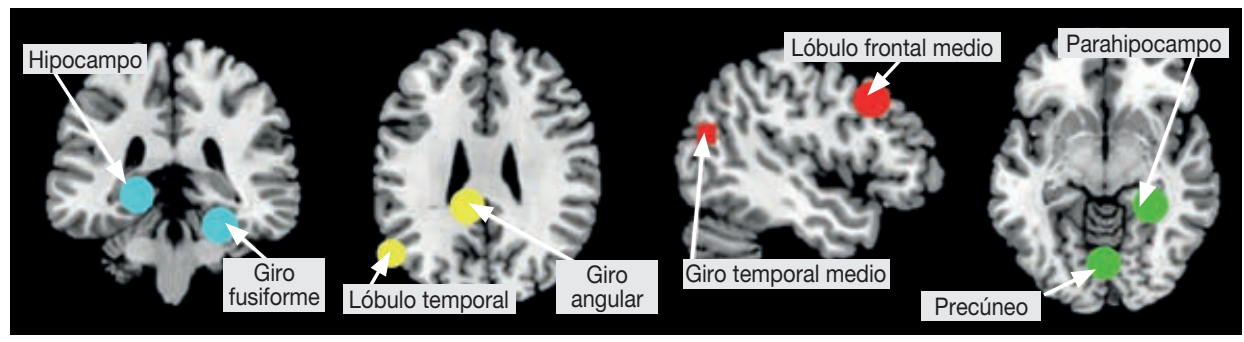

Azul claro: vóxeles que forman parte exclusivamente de la ROI de codificación de la memoria. Amarillo: vóxeles que forman parte exclusivamente de la ROI de reconocimiento.

Rojo: vóxeles relacionados con la memoria de trabajo.

Verde: vóxeles que forman parte principalmente del procesamiento de la recuperación de la memoria.

Las coordenadas corresponden al espacio estándar del Instituto Neurológico de Montreal (MNI). 


\section{Discusión}

En los últimos años, la competencia en el sector del e-commerce ha crecido de forma exponencial, y los minoristas electrónicos buscan estrategias para diseñar sitios web que fomenten de forma más eficaz la compra de productos online. Estudios anteriores de la literatura de marketing interactivo concluyeron que las características de los sitios web constituyen los principales impulsores de las actitudes y los comportamientos de los consumidores online. Aunque los dos entornos web más utilizados, el hedónico y el utilitario, han sido ampliamente definidos, no hay conclusiones unánimes sobre sus efectos en el recuerdo por parte del consumidor. En nuestra investigación se utilizó por primera vez la neuroimagen para identificar el origen neural de la evaluación y recuerdo de estas dos tipologías de sitios web. Los resultados comportamentales revelan que los consumidores revelan significativamente una mayor precisión a la hora de identificar adecuadamente entornos webs hedónicos previamente vistos en relación con los entornos utilitarios. De forma crucial, esta investigación esclarece el origen neuropsicológico del mayor recuerdo de entornos de compra online hedónicos. Concretamente, confirmamos que, mientras que la recuperación de los diseños hedónicos activa áreas cerebrales relacionadas con la codificación de la memoria, el reconocimiento y la memoria de trabajo, los sitios web utilitarios solo activan redes cerebrales relacionadas con la memoria de trabajo. En conjunto, estos resultados aportan información crucial sobre la conexión de los datos neuropsicológicos con el comportamiento del consumidor online en el mundo real, y pueden ayudar al diseño de entornos web eficientes acorde al tipo de producto que se desea comercializar.

Por un lado, los datos derivados de cuestionarios revelan que los participantes recuerdan mejor los sitios web hedónicos como más eficaces. Estos datos se alinean con las conclusiones de los estudios de Rosen y Purinton (2004) y Hausman y Siekpe (2009), quienes afirmaron que los entornos web hedónicos Ilaman más la atención, facilitan la usabilidad en la compra y mejoran el compromiso del consumidor. Además, confirman los resultados de estudios tangenciales previos que concluían que los entornos y mensajes emocionales y de relevancia para el individuo (como los hedónicos) tienen mayor facilidad para ser recordados (Panda et al., 2013).

En nuestro estudio, fuimos un paso más allá e identificamos los mecanismos neuronales asociados a ese mayor recuerdo de los sitios web hedónicos. En concreto, y en consonancia con las hipótesis propuestas, concluimos que los diseños hedónicos activan áreas ampliamente implicadas con la memoria de trabajo (lóbulo temporal y frontal medio), la codificación de la memoria (hipocampo y lóbulo frontal superior), recuperación de la memoria (precúneo y giro parahipocampo) y reconocimiento (giro fusiforme y lóbulo temporal inferior y medio). La comparación de entornos utilitarios vs. hedónicos, no obstante, solo provocó activaciones superiores en áreas implicadas con la memoria de trabajo, esto es, la memoria a corto plazo que se ocupa del procesamiento perceptivo y lingüístico inmediato y consciente. En otras palabras, nuestros resultados confirman que los entornos hedónicos 
se recuerdan mejor no solo debido a que implican a activaciones relacionadas con la recuperación rápida y fugaz de la memoria (como ocurre también en entornos utilitarios), sino porque, además, inducen activaciones cerebrales relacionadas con la grabación de información relevante en nuestro sistema de memoria, así como la recuperación de la misma mediante el recuerdo y el reconocimiento.

Estos resultados se alinean con investigaciones previas de la neurociencia del consumidor que analizan entornos hedónicos y utilitarios desde diferentes perspectivas. Por ejemplo, Schaefer y Rotte (2007) descubrieron que las marcas experienciales de lujo (similares a los sitios web hedónicos) provocan la activación de áreas cerebrales relacionadas con la recompensa (como la corteza ventromedial y el precúneo). En una línea similar, Goodman et al. (2017) analizaron las motivaciones hedónicas y utilitarias de los consumidores a través de fMRI y concluyeron que la motivación experiencial (similar a los sitios web hedónicos) comprometió áreas asociadas con la emoción y la autorreflexión, como la corteza cingulada posterior y el giro frontal inferior. Del mismo modo, investigaciones posteriores descubrieron que la corteza ventromedial frontal y la circunvolución angular son responsables del procesamiento de los estímulos emocionalmente salientes (lo llaman evaluación «en caliente»), como los diseños hedónicos. Incluso investigaciones anteriores en el campo de la publicidad concluyeron que los mensajes emocionales y de autorrelevancia son capaces de implicar áreas cerebrales asociadas con el valor, la memoria de trabajo y la codificación de la memoria.

Teóricamente, estos resultados arrojan luz sobre la falta de unanimidad en torno a la evaluación de la eficacia en los sitios web hedónicos y utilitarios. En concreto, no solo explicamos las diferencias en el procesamiento neural e inconsciente de ambos sitios web, sino que también arrojamos luz sobre el procesamiento psicológico que podría ser responsable de la mayor eficacia de los sitios web hedónicos y utilitarios. Los hallazgos difieren de los estudios anteriores que evalúan los correlatos neurales de los diseños funcionales y experienciales y las motivaciones. Couwenberg et al. (2017), por ejemplo, analizaron los mecanismos neuronales que subyacen al procesamiento de mensajes que enfatizan los beneficios funcionales y experienciales del consumo de un producto, pero sin llevar a cabo una manipulación estricta, controlada y probada previamente de los entornos funcionales (similares a los utilitarios) y experienciales (similares a los hedónicos), y en el contexto publicitario (y no de compra online). Además, Motoki et al. (2019) y Goodman et al. (2017) analizaron los correlatos neuronales de los productos hedónicos y utilitarios y las motivaciones de compra, respectivamente, dejando de lado la importancia del entorno de presentación del producto (es decir, el sitio web hedónico o utilitario) en la evaluación del proceso de compra. En este caso, controlamos el tipo de producto y la motivación para que los mecanismos neuronales estén dirigidos exclusivamente por la condición de interés: el entorno web.

Nuestro estudio constituye, además, un paso más en la identificación de los mecanismos neurales derivados del procesamiento y recuerdo de productos en diferentes entornos web. Estudios anteriores de neurociencia del consumidor evaluaron el procesamiento neural de los pagos electrónicos (Casado-Aranda et al., 2018), los correlatos neurales de 
las señales de confianza online (Casado-Aranda et al., 2019), el procesamiento neural de las presentaciones eficaces de productos (Jai et al., 2021) y la previsión neural del éxito del marketing viral (Motoki et al., 2021). Además, la presente investigación constituye la primera en utilizar una combinación de datos autoinformados y neuronales derivados de las evaluaciones de los sitios web online para predecir los comportamientos online. La mayor capacidad de predicción de las respuestas neuronales para el comportamiento de compra online en entornos hedónicos puede sugerir que el proceso de toma de decisiones en los sitios web hedónicos es interno y de naturaleza más inconsciente e interna, en comparación con el de los sitios web utilitarios.

Los comerciantes electrónicos podrían utilizar nuestros resultados en su gestión en un doble sentido. En primer lugar, el mayor procesamiento neuronal relacionado con el recuerdo, recuperación de la memoria y la relevancia que se deriva de los sitios web hedónicos podría sugerir el uso de dicho entorno web para aquellas estrategias de producto que deseen transmitir valores y atributos relacionados con la emoción, la recompensa y la creación de mayor memoria y reconocimiento (por ejemplo, productos hedónicos, como chocolates o coches de lujo). En cambio, el procesamiento deliberativo y lingüístico propio de los sitios web utilitarios podría sugerir el empleo de elementos utilitarios para aquellos productos que requieren una comprensión específica, concreta y planificada, como los productos preeminentemente utilitarios (por ejemplo, la calculadora o el microondas).

En segundo lugar, los minoristas electrónicos deberían utilizar los resultados neuronales como una nueva metodología para analizar la eficacia de los sitios web. Si las empresas son capaces de crear entornos web hedónicos que provoquen activaciones cerebrales relacionadas con la codificación y recuperación de la memoria y la emoción, y sitios web utilitarios que promuevan un fuerte procesamiento de control cognitivo y la identificación de objetos, entonces podrían considerarse eficaces sin tener que recurrir a herramientas sesgadas y a menudo poco concluyentes como el autoinforme.

\section{Limitaciones, futuras investigaciones}

El presente manuscrito tiene varias limitaciones que deben considerarse. En primer lugar, controlamos el tipo de producto que comprar en la web (ni hedónico ni utilitario) para que no afecte al proceso de evaluación del entorno web (Baltas et al., 2017). Sin embargo, futuras investigaciones deberían corroborar nuestros resultados neuronales en la presentación de productos preeminentemente hedónicos y utilitarios, ya que, en estos casos, la capacidad predictiva de los datos neuronales puede ser más fuerte. A pesar de haber controlado minuciosamente las manipulaciones de los sitios web hedónicos y utilitarios (para evitar la presencia de factores de confusión), estos entornos de compra pueden diferir de los diseños reales en línea; por lo tanto, la artificialidad del diseño experimental y del contexto de fMRI puede constituir un inconveniente. Aunque los estudios anteriores han utilizado el recuerdo 
como una medida conductual (Couwenberg et al., 2017), las investigaciones futuras podrían asociar los datos neuronales con las compras reales en el mercado online. Además, los estudios prospectivos son una buena oportunidad para utilizar otras técnicas de psicofisiología (como la conductancia de la piel, la frecuencia cardíaca o la electromiografía) para ofrecer nuevos conocimientos psicológicos y corroborar los resultados de la presente investigación.

En conclusión, los hallazgos actuales mostraron, por primera vez, el origen neural del procesamiento de diseños web hedónicos y utilitarios. Curiosamente, esta investigación constituye una primicia al demostrar que los datos neuronales sobre las características de los sitios web ayudan a predecir el comportamiento de los consumidores en línea, más allá de la eficacia percibida de los sitios web comunicada por los consumidores. Por lo tanto, hemos demostrado la necesidad de complementar las técnicas tradicionales de autoinforme con datos de neuroimagen más objetivos y en cada momento para explicar el comportamiento del consumidor online.

\section{Conclusiones}

A pesar de la gran cantidad de literatura que profundiza en los efectos de los entornos webs sobre la experiencia y la percepción del consumidor, sorprene que la mayoría de los estudios no han comparado los efectos de la visualización de entornos de compra hedónicos y utilitarios. Este es el primer estudio que aplica un marco multimetodológico para abordar dicha laguna de investigación. Avanza que el mayor recuerdo ocasionado por entornos de compra hedónicos tiene su origen en mecanismos psicológicos asociados al valor, la autoimportancia y la codificación y recuperación de la memoria. Entornos utilitarios, por su parte, provocan solo mayores activaciones cerebrales vinculadas al procesamiento deliberativo y memoria de trabajo. Por lo tanto, este estudio permite comprender el origen de las actitudes y recuerdo de entornos webs hedónicos y utilitarios.

\section{Referencias bibliográficas}

Baltas, G., Kokkinaki, F. y Loukopoulou, A. (2017). Does variety seeking vary between hedonic and utilitarian products? The role of attribute type. Journal of Consumer Behaviour, 16(6), e1- e12. https://doi. org/10.1002/cb.1649
Bilgihan, A. y Bujisic, M. (2015). The effect of website features in online relationship marketing: A case of online hotel booking. Electronic Commerce Research and Applications, 14(4), 222-232. https://doi. org/10.1016/j.elerap.2014.09.001 
Borghesani, V., Narvid, J., Battistella, G., Shwe, W., Watson, C., Binney, R. J., Sturm, V., Miller, Z., Mandelli, M. L., Miller, B. y Gorno-Tempini, M. L. (2019). "Looks familiar, but I do not know who she is": The role of the anterior right temporal lobe in famous face recognition. Cortex, 115, 72-85. https://doi.org/10.1016/j. cortex.2019.01.006

Casado-Aranda, L.-A., Dimoka, A. y SánchezFernández, J. (2019). ConsumerProcessing of Online Trust Signals: A Neuroimaging Study. Journal of Interactive Marketing, 47, 159-180. https://doi.org/10.1016/j. intmar.2019.02.006

Casado-Aranda, L.-A., Sánchez-Fernández, J. y Montoro-Ríos, F. J. (2018). How Consumers Process Online Privacy, Financial, and Performance Risks: An fMRI Study. CyberPsychology, Behavior \& Social Networking, 21(9), 556-562. Business Source Complete. https://doi.org/10.1089/ cyber.2018.0196

Couwenberg, L. E., Boksem, M. A. S., Dietvorst, R. C., Worm, L., Verbeke, W. J. M. I. y Smidts, A. (2017). Neural responses to functional and experiential ad appeals: Explaining ad effectiveness. International Journal of Research in Marketing, 34(2), 355-366. https://doi.org/10.1016/j. ijresmar.2016.10.005

Dimoka, A. (2010). What does the brain tell us about trust and distrust? Evidence from a functional neuroimaging study. Mis Quarterly, 2(34), 373-396.

eMarketer. (2021). Global ecommerce update 2021. https://www.emarketer.com/content/ global-ecommerce-update-2021

Falk, E. B., Rameson, L., Berkman, E. T., Liao, B., Kang, Y., Inagaki, T. K. y Lieberman, M. D. (2010). The neural correlates of persuasion: A common network across cultures and media. Journal of Cognitive Neuroscience, 22(11), 2.447-2.459.
Goodman, A. M., Wang, Y., Kwon, W.-S., Byun, S.-E., Katz, J. S. y Deshpande, G. (2017). Neural correlates of consumer buying motivations: A $7 \mathrm{~T}$ functional magnetic resonance imaging (fMRI) study. Frontiers in Neuroscience, 11. https://doi. org/10.3389/fnins.2017.00512

Guerrero Medina, C. A., Martínez-Fiestas, M., Casado Aranda, L. A. y Sánchez-Fernández, J. (2021). Is it an error to communicate CSR Strategies? Neural differences among consumers when processing CSR messages. Journal of Business Research, 126, 99-112. https://doi.org/10.1016/j. jbusres.2020.12.044

Hausman, A. V. y Siekpe, J. S. (2009). The effect of web interface features on consumer online purchase intentions. Journal of Business Research, 62(1), 5-13. https:// doi.org/10.1016/j.jbusres.2008.01.018

Hubert, M., Hubert, M., Linzmajer, M., Riedl, R., Kenning, P. y\& Hubert, M. (2018). Trust me if you can - neurophysiological insights on the influence of consumer impulsiveness on trustworthiness evaluations in online settings. European Journal of Marketing, 52(1/2), 118-146. https://doi. org/10.1108/EJM-12-2016-0870

Jai, T.-M. (Catherine), Fang, D., Bao, F. S., James, R. N., Chen, T. y Cai, W. (2021). Seeing It Is Like Touching It: Unraveling the Effective Product Presentations on Online Apparel Purchase Decisions and Brain Activity (An fMRI Study). Journal of Interactive Marketing, 53, 66-79. https://doi. org/10.1016/j.intmar.2020.04.005

Johnson, E. L., Adams, J. N., Solbakk, A.-K., Endestad, T., Larsson, P. G., Ivanovic, J., Meling, T. R., Lin, J. J. y Knight, R. T. (2018). Dynamic frontotemporal systems process space and time in working memory. PLOS Biology, 16(3), e2004274. https://doi. org/10.1371/journal.pbio.2004274

Li, J., Abbasi, A., Cheema, A. y Abraham, L. B. (2020). Path to Purpose? How Online 
Customer Journeys Differ for Hedonic Versus Utilitarian Purchases. Journal of Marketing, 84(4), 127-146. https://doi. org/10.1177/0022242920911628

Liang, T.-P. y Lai, H.-J. (2002). Effect of store design on consumer purchases: An empirical study of on-line bookstores. Information \& Management, 39(6), 431-444. https:// doi.org/10.1016/S0378-7206(01)00129-X

Mazaheri, E., Richard, M. y Laroche, M. (2012). The role of emotions in online consumer behavior: A comparison of search, experience, and credence services. Journal of Services Marketing, 26(7), 535-550. https:// doi.org/10.1108/08876041211266503

Moon, M. A., Khalid, M. J., Awan, H. M., Attiq. S., Rasool, H. y Kiran, M. (2017). Consumer's perceptions of website's utilitarian and hedonic attributes and online purchase intentions: A cognitive-affective attitude approach. Spanish Journal of Marketing - ESIC, 21(2), 73-88. https://doi. org/10.1016/j.sjme.2017.07.001

Motoki, K., Suzuki, S., Kawashima, R. y Sugiura, M. (2020). A Combination of selfreported data and social-related neural measures forecasts viral marketing success on social media. Journal of Interactive Marketing, 52, 99-117. https://doi. org/10.1016/j.intmar.2020.06.003

Panda, T. K., Panda, T. K. y Mishra, K. (2013). Does emotional appeal work in advertising? The rationality behind using emotional appeal to create favorable brand attitude. IUP Journal of Brand Management, 10(2), 7.

Rose, S., Hair, N. y Clark, M. (2011). Online customer experience: A review of the businessto-consumer online purchase context: online customer experience. International Journal of Management Reviews, 13(1), 24-39. https:// doi.org/10.1111/j.1468-2370.2010.00280.x

Rosen, D. E. y Purinton, E. (2004). Website design: Viewing the web as a cognitive landscape. Journal of Business Research, 57(7), 787-794. https://doi.org/10.1016/ S0148-2963(02)00353-3

Schaefer, M. y Rotte, M. (2007). Thinking on luxury or pragmatic brand products: Brain responses to different categories of culturally based brands. Brain Research, 1165, 98-104. https://doi.org/10.1016/j. brainres.2007.06.038

Statista. (2021). Global digital buyer reach 2021. https://www.statista.com/statistics/261676/ digital-buyer-penetration-worldwide/

Tsukiura, T. y Cabeza, R. (2011). Remembering beauty: Roles of orbitofrontal and hippocampal regions in successful memory encoding of attractive faces. Neurolmage, 54(1), 653-660. https://doi.org/10.1016/j. neuroimage.2010.07.046

Yarkoni, T., Poldrack, R. A., Nichols, T. E., Van Essen, D. C. y Wager, T. D. (2011). Largescale automated synthesis of human functional neuroimaging data. Nature Methods, 8(8), 665-670. https://doi.org/10.1038/ nmeth.1635 